\title{
Optical Glass Substrates Forming Processes Using Pulsed Ultraviolet Laser Micromilling Technology
}

\author{
Wen-Tse Hsiao ${ }^{*}$, Chih-Chung Yang ${ }^{*}$, Kuo-Cheng Huang ${ }^{*}$, Chien-Kai Chung ${ }^{*}$, Shih-Feng Tseng ${ }^{*}$, Donyau Chiang ${ }^{*}$ and Ming- \\ Fei Chen $^{* *}$ \\ * Instrument Technology Research Center, National Applied Research Laboratories, Taiwan, No.20, \\ R\&D Rd. VI, Hsinchu Science Park, Hsinchu, 30076, Taiwan \\ E-mail: wentse@narlabs.org.tw \\ ** Department of Mechatronics Engineering, National Changhua University of Education, Taiwan, \\ No.1, Jin-De Road, Changhua City, 50007, Taiwan
}

\begin{abstract}
Glass exhibits excellent optical transmittance in the visible spectrum (400-700 nm). Therefore, it is widely used in optical systems such as laser projection and light delivering. In this study, an ultraviolet laser system was combined with a high-speed galvanometric scanner unit to mill optical glass materials. First, a numerical simulation approach was used to evaluate the effect of the bite distance of milling. After the laser milling of optical glass materials (sodalime and B270 glass), the surface morphology, surface roughness, and cross-section quality were analyzed using a three-dimensional confocal laser scanning microscope to evaluate the effects of milling parameters on optical glass materials.
\end{abstract}

DOI: $10.2961 / \mathrm{jlmn} .2016 .01 .0006$

Keywords: high-speed galvanometric scanner, laser milling, optical glass, numerical simulation, surface roughness.

\section{Introduction}

With the increasing popularity of smart phones, every smartphone is now equipped with various types of functions. Because of the development of miniature projection technology from large screen projection technology, the portable electronic has become a highly anticipated new display product. However, projectors were mainly used in the past for meetings, teaching, and home theaters. The integration of the opto-electro-mechanical system (OEMS) technology in products has led the development of smart products. These products are light, thin, consume less power, and portable, and the concept of miniature projector has become a major star in the electronics industry. The commonly used methods for microstructure fabrication on optical glass substrates include (1) diamond wheel grinding, (2) wet etching, (3) ultra-precision machining, and (4) molding. However, the aforementioned methods require multiple steps and chemicals. In addition, chemicals required for wet etching are hazardous for the environment.

Laser processing is a noncontact processing mode and can be combined with a galvanometer scanning unit for achieving high speed and high precision processing. By using a special focusing lens, the laser spot diameter can be reduced to several tens of micrometers. Photo fabricationbased lasers have become a niche market.

Dunsky [1] described the laser manufacturing technology that is used in industries and that represents the overall market demand for optical and electronic fields. The application of laser technology can be divided into six processes: (A) semiconductor wafer fabrication: front-end (photolithography, mask production, and optical detection), (B) semiconductor testing and assembly process: back-end (wafer cutting and engraving), (C) inner structure and ad- vanced packaging technology process (blind hole, drilling, and laser direct imaging), (D) flat panel display manufacturing process (transparent conductive oxide films patterning, low temperature annealing, re-crystallization, and glass cutting), (E) micromachining process (sapphire-based, silicon-based, and SiC-based materials), and (F) other processes (solar materials and optical storage materials). Gamaly et al. [2] proposed the removal mechanism and threshold evaluation for various metal and dielectric materials by using a femtosecond pulse laser. The results indicated that the removal thresholds of copper and gold materials were 0.51 and $0.5 \mathrm{~J} / \mathrm{cm}^{2}$, respectively. In addition, three wavelengths $(825,526$, and $1053 \mathrm{~nm})$ of a picosecond laser were used to evaluate the material ablation threshold of silica. The results indicated that the thresholds were 1.84 , 1.17 , and $2.0 \mathrm{~J} / \mathrm{cm}^{2}$ at 825,526 , and $1053 \mathrm{~nm}$, respectively. Florea [3] used a femtosecond laser to fabricate the lines and curves waveguide structure on silicate glass and the Fabry-Pérot resonator to measure the waveguide loss. In addition, periodic grating was annealed for different durations. The results indicated that the diffraction efficiency was approximately $8 \%$. Zheng and Hee et al. [4,5] used a femtosecond laser to produce a periodic structure on fused silica and crystalline silicon materials by using an interference lithography method. Zheng et al. [4] used a Ppolarization femtosecond laser to fabricate a crack-free periodic grating structure with a line width of $20 \mu \mathrm{m}$. In this study, the microstructure in the vertical direction had significant polarization. Hee et al. [5] used four types of polarization modes to fabricate a periodic microstructure. The results indicated that the polarization mode affected the grating depth, surface roughness, and removal threshold. Florea [6], Cho [7], and Ams [8] et al. used the femtosec- 
ond laser pulse properties to produce a two-dimensional structure of the interior of the glass substrate. Oliveira [9] et al. used a range of laser pulse energy, from 0.5 to $2 \mathrm{~J} / \mathrm{cm}^{2}$, to produce micro and nano-periodic and non-periodic microstructures of titanium. The results indicated that the shock wave diffused from the center to outwards. In addition, scan speeds affected the periodic structure. Therefore, the laser energy is lower than the ablation threshold that could generate various periodic micro and nanostructures on titanium substrates. In addition, the manufacturing of micro and nanostructures is based on the demand of polymer materials such as PDMS and PMMA [10-12].

In this study, an optical glass substrate with a microstructure was fabricated by combining an ultraviolet (UV) laser with a high-speed galvanometric scanning unit and milling. The surface morphology, surface roughness, and cross-section quality were analyzed using a threedimensional confocal laser-scanning microscope to evaluate the effects of milling parameters on optical glass materials

\section{Ultraviolet laser micromilling system, milling theory, and milling path planning}

\subsection{UV laser micromilling system}

Diode-pumped solid-state (DPSS) Nd: $\mathrm{YVO}_{4} \mathrm{UV}$ laser (COHERENT, Inc., AVIA 355-14) (wavelength $=355 \mathrm{~nm}$, maximum output power level $=14 \mathrm{~W}$, transverse mode $=$ $\mathrm{TEM}_{00}$, output beam diameter $=3.5 \mathrm{~mm}$ ) was combined with a high-speed galvanometer system (Raylase AG model SS-15) in which a focus shifter was used to adjust the focus range in the Z-direction from +15 to $-15 \mathrm{~mm}$ and $\mathrm{x}-$ $y$ axes moving stage. The telecentric focusing lens with a focal length of $110 \mathrm{~mm}$ and a scanning area of $40 \mathrm{~mm}^{2}$ was used for drilling, and the operation pulse repetition frequency (PRF; $1-400 \mathrm{kHz}$ ) was used for laser trepanning for drilling through holes on polycrystalline silicon substrates. Table 1 summarizes the complete specifications of the DPSS laser milling system.

Table 1 Specification of DPSS laser milling system.

\begin{tabular}{lc}
\hline Item & Parameter \\
\hline Wavelength $(\mathrm{nm})$ & 355 \\
Output power (Watt) & $>14$ \\
Mode & $\mathrm{TEM}_{00}$ \\
Pulse repetition frequency $(\mathrm{kHz})$ & $1-400$ \\
Pulse width $(\mathrm{ns})$ & 28 \\
Output beam size $(\mathrm{mm})$ & 3.5 \\
Focal length of scan lens $(\mathrm{mm})$ & 110 \\
Scan field $\left(\mathrm{mm}^{2}\right)$ & 60 \\
Focus beam spot size $(\mu \mathrm{m})$ & 30 \\
\hline
\end{tabular}

\subsection{Laser micro-milling principle}

Figure 1 (a) shows the schematic of the laser milling of a glass substrate. Figure 1 (b) shows the laser beam overlapping rate of milling. The overlapping rate affected the milled edge quality, surface roughness, and removal rate. The factors that influence the overlapping rate include (1) the scan speed of the galvanometric unit, (2) PRF, (3) focused beam diameter, and (4) the bite size of each overlapped laser beam.
The bite size and overlapping rate can be calculated using Eq. (1) and (2), respectively, as follows:

$$
\begin{aligned}
& B_{s}=\frac{V}{f} \\
& O_{R}(\%)=\frac{b-B_{s}}{b} \times 100
\end{aligned}
$$

where $B_{s}$ is the bite size ( $\left.\mathrm{mm}\right), V$ is the scan speed $(\mathrm{mm} / \mathrm{s}), f$ is the PRF $(\mathrm{Hz}), O_{R}$ is the overlapping rate, and $b$ is the laser beam spot size ( $\mathrm{mm})$.
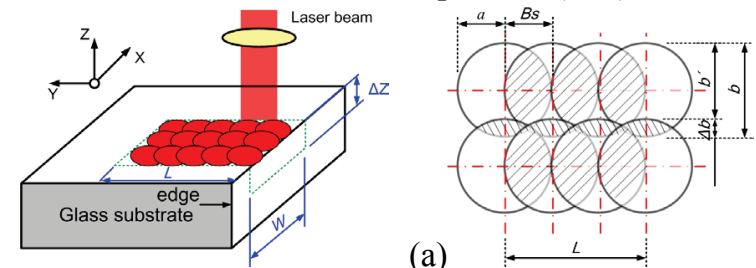

Fig. 1 (a) Schematic of the laser milling of a glass substrate, (b) laser beam bite size for a two-dimensional milling path.

For evaluating the milling time, the horizontal axis $\left(N_{x}\right)$ can be calculated using Eq. (3) as follows:

$$
N_{x}=\frac{L}{B_{s}}=\frac{L \cdot f}{V}
$$

where $L$ is the length of the milling region ( $\mathrm{mm})$ and $a$ is the non overlapping distance $(\mathrm{mm})$. When the overlapping distance is half of the beam size, Eq. (3) can be rewritten as Eq. (4) as follows:

$$
N_{x}=\frac{L}{a}
$$

The vertical axis $\left(N_{y}\right)$ can be calculated using Eq. (5) as follows:

$$
N_{y}=\frac{w}{b^{\prime}}+1
$$

where $w$ is the width of the milling region $(\mathrm{mm}), b^{\prime}$ is the non overlapping distance along the y-axis, and $\Delta b$ is the bite size of milling along the $y$-axis that can be calculated using Eq. (6), as follows:

$$
b^{\prime}=b-\Delta b
$$

For calculating the milling area and total milling time of laser micromilling, Eqs. (4) and (5) are multiplied, and the desired action time $(t)$ for each pulsed laser source is considered. The total time can be then be obtained by milling $\left(T_{\text {process }} ;\right.$ Eq. (7)), as follows:

$$
\begin{gathered}
T_{\text {process }}=N_{x} \cdot N_{y} \cdot t \\
=\frac{L}{a} \cdot\left(\frac{w}{b^{\prime}}+1\right) \cdot t \\
=\frac{L}{a} \cdot\left(\frac{w}{(b-\Delta b)}+1\right) \cdot t
\end{gathered}
$$

where $t$ is $1 / f$. Now, Eq. (7) can be modified to Eq. (8) as follows:

$$
T_{\text {process }}=\frac{L}{a} \cdot\left(\frac{w}{(b-\Delta b)}+1\right) \cdot \frac{1}{f}
$$

If the volume of the laser milling zone volume is the product of length $(\mathrm{L})$, width $(\mathrm{w})$, and depth $(\Delta z), \rho$ is the density of the material $\left(\mathrm{kg} / \mathrm{m}^{3}\right)$. The material removal rate 
can be calculated using Eq. (9) as follows [13]:

$$
\operatorname{MRR}=\frac{\rho \cdot L \cdot w \cdot \Delta z}{T_{\text {process }}}
$$

\subsection{Laser micromilling path planning and experi- mental materials}

Figure 2 shows the experimental setup. In this study, two types of optical glasses with thicknesses of $3 \mathrm{~mm}$ and $10 \mathrm{~mm}$, respectively, were used for milling. To evaluate the geometric dimension and surface quality of the milling region, the milling area was fixed to at $3 \times 3 \mathrm{~mm}$ (Fig. 2 (a)). Figure 2 (b) shows the schematic of the measurement regions of depth and surface roughness, and Fig. 2 (c) shows the schematic of the laser milling path planning. Each milling area $\left(3 \mathrm{~mm}^{2}\right)$ at intervals of $20 \mu \mathrm{m}$ in horizontal and vertical directions was used for evaluation. Moreover, the milling angles of the horizontal path were set to $0^{\circ}$ and $30^{\circ}$, and those of the vertical path were set to $60^{\circ}$ and $90^{\circ}$.

Tables 2 and 3 show the laser milling parameters for the milling of soda-lime glass and B270 glass, respectively.

(a) Milling regions

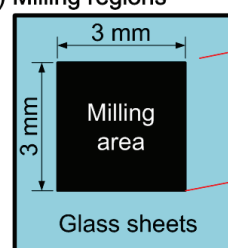

(c) Milling path planning

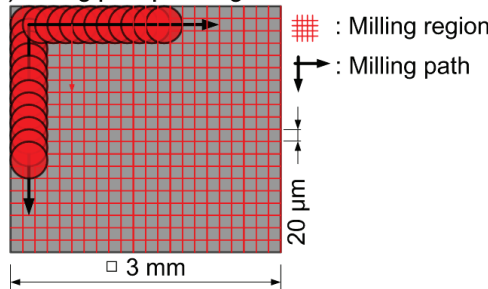

Fig. 2 Experimental setup; (a) laser milling region, (b) depth and roughness measurement regions, (c) laser milling path planning, and (d) laser milling path angle.

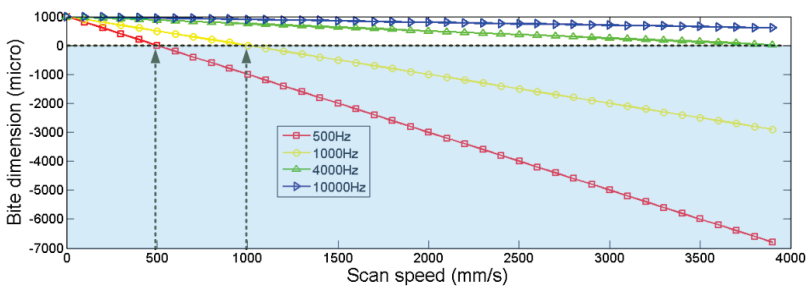

Non-continuous region

Fig. 3 Relationship between scan speed and bite distance.

Table 2 Experimental parameters of laser milling of B270 glass substrates.

\begin{tabular}{ll}
\hline Item & Parameter \\
\hline Laser power $(\mathrm{W})$ & 14 \\
Pulse repetition frequency $(\mathrm{kHz})$ & 100 \\
Scan speed $(\mathrm{mm} / \mathrm{s})$ & $500,800,1000$ \\
Number of milling passes & $3,5,7$ \\
Milling angle (degree) & $0,30,60,90$ \\
\hline
\end{tabular}

Table 3 Experimental parameters of laser milling of soda-lime glass substrates.

\begin{tabular}{ll}
\hline Item & Parameter \\
\hline Laser power $(\mathrm{W})$ & 14 \\
Pulse repetition frequency $(\mathrm{kHz})$ & 70 \\
Scan speed $(\mathrm{mm} / \mathrm{s})$ & 2000 \\
Number of milling passes & $1,5,10,15,20,30$ \\
\hline
\end{tabular}

\section{Results and discussion}

\subsection{Simulation of laser spot bite size and the overlap-} ping rate

Figure 3 shows the simulation results of the relationship between the scan speed and bite size at various PRFs. The beam overlapping rate was greater than $0 \%$ and the PRF was set to 0.5 and $1 \mathrm{kHz}$. Therefore, the scan speed was expected to be lower than 500 and $1000 \mathrm{~mm} / \mathrm{s}$, respectively According to the simulation results, the scan speed increased with an increase in the PRF. To obtain optimum overlapping, the ratio of PRF to scan speed should be less than one.

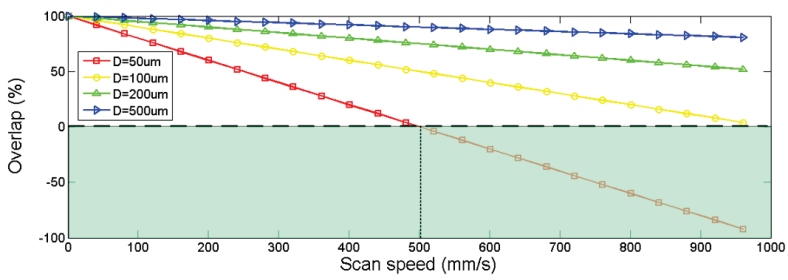

Non-continuous region

(a)

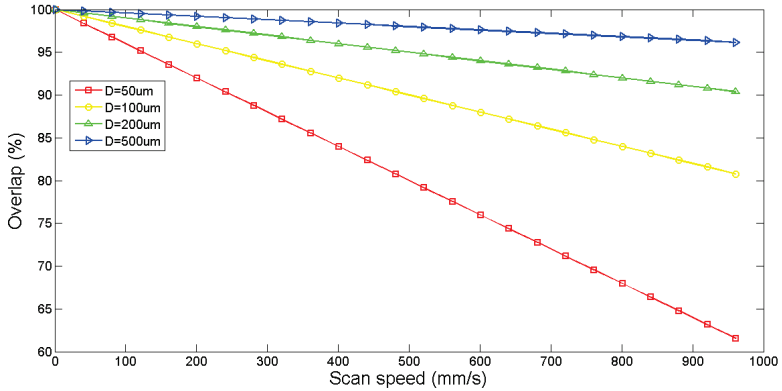

(b)

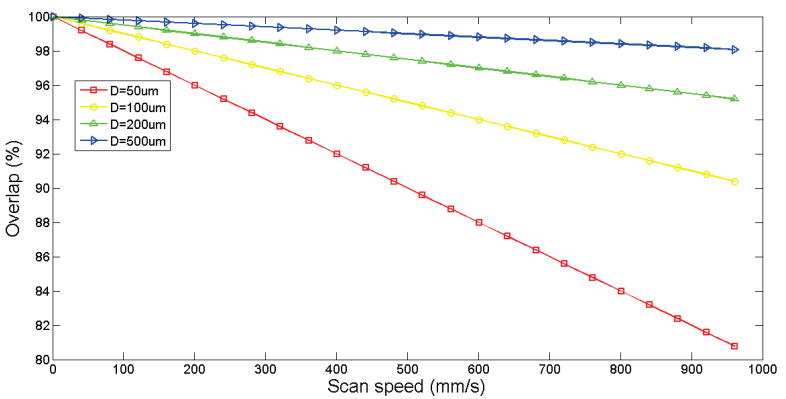

Fig. 4 Relationship between scan speed and overlapping rate at various beam sizes and PRFs; (a) $10 \mathrm{kHz}$, (b) $50 \mathrm{kHz}$, and (c) 100 $\mathrm{kHz}$.

According to the simulation results, the spot diameter at different $(50 \mu \mathrm{m}, 100 \mu \mathrm{m}, 200 \mu \mathrm{m}, 500 \mu \mathrm{m})$ condition, when the laser pulse is repeated with the frequency of 50 $\mathrm{kHz} 100 \mathrm{kHz}$, and a scanning speed of $1000 \mathrm{~mm} / \mathrm{s}$, the rate of its spot overlap more than $60 \%$, as the spot diameter becomes larger process, which can be obtained with a high overlap rate of the scanning speed, which can increase the 
process speed. When the laser PRF is $10 \mathrm{kHz}$ and the spot diameter is $50 \mu \mathrm{m}$, the scan speed is greater than $500 \mathrm{~mm} / \mathrm{s}$, which is lower than the spot overlap ratio of $0 \%$.

\subsection{Depth and surface roughness evaluation of laser- milled soda-lime glass $(t=3 \mathrm{~mm})$}

Figure 5 (a) shows a plot of milling depth versus number of milling passes of soda-lime glass $(t=3 \mathrm{~mm})$. The laser power, PRF, and scan speed were set at $14 \mathrm{~W}, 70 \mathrm{kHz}$, and $2000 \mathrm{~mm} / \mathrm{s}$, respectively. As shown, the milling depth increased with an increase in the number of milling passes. After milling ten times, the milling depth increased to 100 $\mu \mathrm{m}$. For measuring the surface roughness, the ISO-4287 standard was used to revolute the milled regions. Figure 5 (b) shows a plot of milled surface roughness versus number of milling passes. To obtain a surface roughness $(R a)$ of more than $10.5 \mu \mathrm{m}$, number of milling passes of two and 30 were required. Figure 5 (c) shows the surface morphology of the milled region recorded using a threedimensional confocal microscope. Each parameter was measured five times to provide data for an average value.

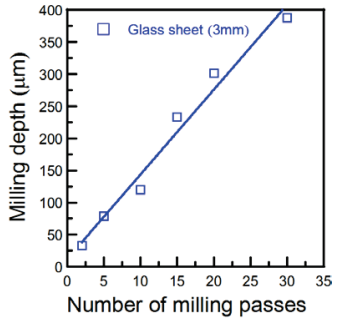

(a)

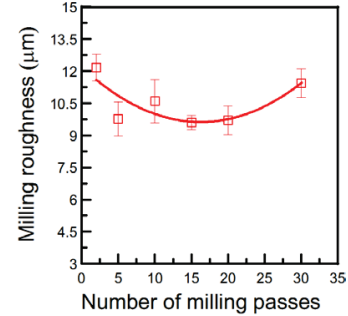

(b)

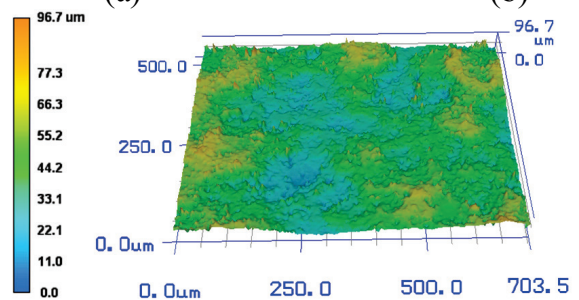

(c)

Fig. 5 Soda-lime glass $(t=3 \mathrm{~mm})$ laser milled revolution; (a) depth, (b) surface roughness, and (c) surface morphology.

\subsection{Depth and surface roughness evaluation of laser milled B270 glass $(t=10 \mathrm{~mm})$}

Figure 6 shows a plot of milling depth versus milling speed at various milling angles on B270 glass $(t=10 \mathrm{~mm})$. The laser power and PRF were set at $14 \mathrm{~W}$ and $100 \mathrm{kHz}$, respectively. Similar to the trend seen for the soda-lime glass, the milling depth increased with an increase in the number of milling passes. To obtain a milling depth of more than $500 \mu \mathrm{m}$, a number of milling passes of more than five was required. A deep microstructure was obtained at a high milling speed. This is because high-speed milling causes the crack phenomenon. In addition, the milling angle did not affect the milling depth. Figure 7 shows a plot of the surface roughness revolution of laser-milled B270 glass at a milling speed of 500, 800, and $1000 \mathrm{~mm} / \mathrm{s}$. The milling roughness increased with an increase in the number of milling passes. After laser milling, the milled surface roughness values ranged from 40 to $180 \mu \mathrm{m}$.

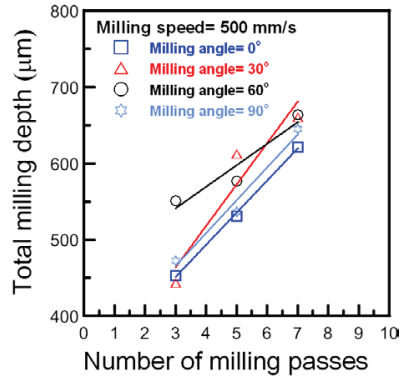

(a)

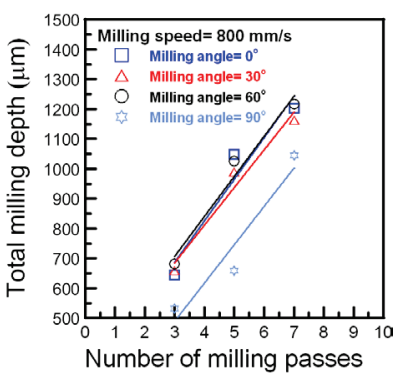

(b)

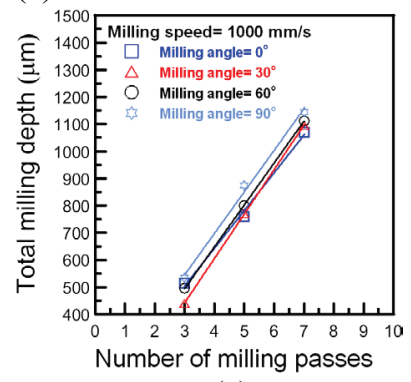

(c)

Fig. 6 Relationship between number of milling passes and milling depth at different milling angle and milling speed.

(a) $500 \mathrm{~mm} / \mathrm{s}$, (b) $800 \mathrm{~mm} / \mathrm{s}$, and (c) $1000 \mathrm{~mm} / \mathrm{s}$.

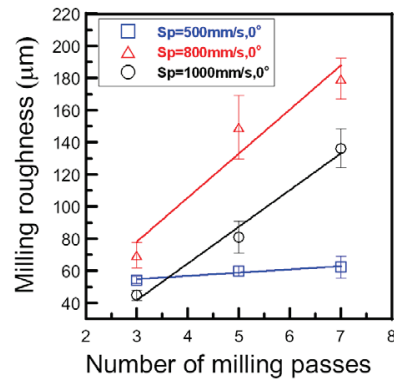

(a)

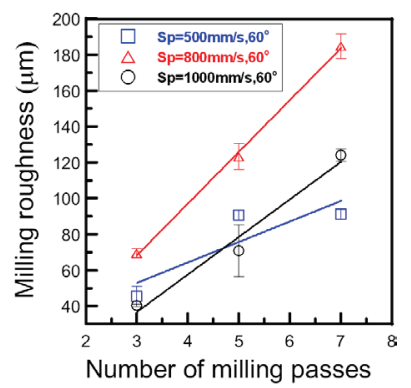

(c)

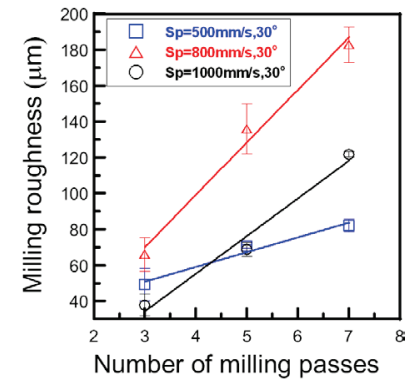

(b)

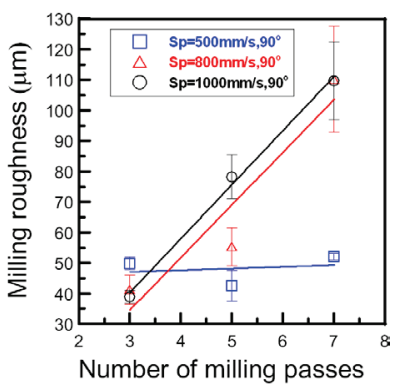

(d)
Fig. 7 Surface roughness revolution of laser-milled B270 glass at various milling angles; (a) $0^{\circ}$, (b) $30^{\circ}$, (c) $60^{\circ}$, and (d) $90^{\circ}$.

\section{Conclusion}

In this study, a UV laser system was successfully combined with a high-speed galvanometric scanner unit for milling optical glass materials (soda-lime and B270 glass). The effects of milling depth, surface roughness, and surface morphology that depended on laser power, scan speed, and scan time and scanning angle, respectively, were discussed. All conclusions of this study are as follows:

(a) In the laser beam overlapping simulation, the scan speed increased with an increase in the PRF. To obtain an 
optimum overlap, the ratio of the PRF to scan speed should be less than one.

(b) A larger laser beam size led to an increase in the milling speed.

(c) The milling depth increased with an increase in the number of milling passes. Moreover, a change in the milling angle did not significantly influence the milling depth.

(d) The crack phenomenon that caused the milling depth increased in high-speed milling.

(e) In the future, the proposed method can be used for the production of optical components and biomedical fluid elements.

\section{Acknowledgment}

The authors thank the National Science Council of Taiwan for financially supporting this research under contract nos. MOST 103-2622-E-492-008 and MOST 103-2221-E-492018.

\section{References}

[1] C.M. Dunsky: Proc. SPIE, Vol. 5713, (2005) p.200.

[2] E.G. Gamaly, A.V. Rode, B. Luther-Davies, V.T. Tikhonchuk: Phys. Plasmas, 9, (2002) 949.

[3] C. Florea: J. Lightwave Technol., 21, (2003) 246.
[4] H.Y. Zheng, W. Zhou, H.X. Qian, T.T. Tan, G.C. Lim: Appl. Surf. Sci., 236, (2004) 114.

[5] C.W. Hee, B.K.A. Ngoi, L.E.N. Lim, K. Venkatakrishnan, W.L. Liang: Opt. Laser Technol., 37, (2005) 93.

[6] C. Florea, J.S. Sanghera, I.D. Aggarwal: Opt. Mater., 30, (2008) 1603.

[7] S.H. Cho, W.S. Chang, J.G. Kim, K.R. Kim, J.W. Hong: Appl. Surf. Sci., 255, (2008) 2069.

[8] M. Ams, G.D. Marshall, P. Dekker, M. Dubov, V. K. Mezentsev, I. Bennion, M.J. Withford: IEEE J. Sel. Top. Quant., 14, (2008) 1370.

[9] V. Oliveira, S. Ausset, R. Vilar: Appl. Surf. Sci., 255, (2009) 7556

[10] S.H. Cho, W.S. Chang, K.R. Kim, J.W. Hong: Opt. Commun., 282, (2009) 1317.

[11]Hiroyuki Mochizuki, Wataru Watanabe, Yasuyuki Ozeki, Kazuyoshi Itoh, Katsumi Matsuda, Satoshi Hirono: Thin Solid Films, 518, (2009) 714

[12]Z. Nie, K.S. Lim, H. Lee, M. Lee, T. Kabayashi: J. Lumin., 131, (2011) 266.

[13] A.N. Samant, N.B. Dahotre: J. Manuf. Proc., 12, (2010) 1.

(Received: May14, 2015, Accepted: January 5, 2016) 